\title{
Pseudoaneurisma postraumático de arteria descendente anterior
}

\section{Post-traumatic false (pseudo) aneurysm of the left anterior descending artery}

Esteban N. Niello', Fabrizio L. Wirz'ㄹ, Juan H. Guiroy ${ }^{3}$

\section{RESUMEN}

El pseudoaneurisma de arteria coronaria es extremadamente raro. Su historia natural es poco conocida y su tratamiento discutido. Se presenta el caso de un paciente con pseudoaneurisma de arteria descendente anterior e infarto anterior del ventrículo izquierdo secundario a un traumatismo penetrante de tórax por herida de arma blanca, tratado con stent recubierto de manera exitosa.

Palabras claves: enfermedad de arteria coronaria, trauma, falso (pseudo) aneurisma coronario.

\section{ABSTRACT}

Coronary pseudo-aneurysm is an extremely rare entity. Its natural history is scarcely known and its treatment is controversial. We report a case of pseudo-aneurysm of the left anterior descending artery associated with an anterior infarct of the left ventricle following a penetrating thoracic trauma, treated with covered stent successfully.

Key words: coronary artery disease, trauma, coronary false aneurysm

Revista Argentina de Cardioangiología Intervencionista 2019;10(1):29-30. DOI: 10.30567/RACI/201901/0029-0030

El pseudoaneurisma coronario es infrecuente y difícil de diferenciar del aneurisma coronario ${ }^{1,2}$. Los casos publicados de pseudoaneurismas coronarios han sido en su mayoría secundarios a procedimientos percutáneos, disecciones espontáneas o traumatismos de tórax, siendo estos últimos extremadamente $\operatorname{raros}^{1-7}$. Las complicaciones más temidas son la ruptura o la embolización del trombo formado en el aneurisma con el consiguiente infarto ${ }^{2}$. El tratamiento es discutido: médico, percutáneo o quirúrgico ${ }^{1-3,8}$. Debido a su rareza, la historia natural de esta entidad es poco conocida.

\section{CASO CLÍNICO}

Hombre de 20 años, con antecedente de traumatismo penetrante en hemitórax izquierdo hace un mes, que requirió toracotomía mínima de urgencia, con colocación de tubo de avenamiento pleural izquierdo por hemoneumotórax en su localidad de origen. Presenta mala evolución, por lo que es derivado a Hospital Público de la capital de la provincia, donde se realiza ECG que evidencia signos compatibles con fibrosis anterior. En ecocardiograma se evidencia derrame pericárdico severo, sin signos de taponamiento cardiaco, con deterioro leve de FEVI (50\%), por trastornos de la motilidad segmentaria (aquinesia de segmentos apicales del septum, pared anterior, lateral e inferior).

Es derivado a nuestro servicio para coronariografía programada que evidencia imagen compatible con pseudoaneurisma en tercio medio de la arteria descendente anterior bilobulado de 3,5 a $4 \mathrm{~mm}$ (Figuras 1 y 2). El resto de los vasos no muestra signos de aterosclerosis. Se realiza en forma programada angioplastia

1. Médico de planta, Instituto Cardiovascular del Chaco

2. Médico de planta, Instituto Cardiovascular del Chaco

3. Jefe de Servicio, Instituto Cardiovascular del Chaco

$\triangle$ Correspondencia: Dr. Esteban Nicolás Niello. Moreno 615, (CP 3400) Corrientes, Corrientes, Argentina.eniello@hotmail.com

Los autores no declaran conflictos de intereses

Recibido: 29/11/2018| Aceptado:08/03/2019 transluminal coronaria a arteria descendente anterior con colocación de un stent coronario cubierto de PTFE marca Be Graft (Bentley) de 3,0 $\mathrm{mm}$ de diámetro por $21 \mathrm{~mm}$ de longitud de manera exitosa (Figura 3), con buena evolución, siendo derivado a su hospital a las $24 \mathrm{hs}$ para controles de rutina.

\section{DISCUSIÓN}

Los aneurismas de arterias coronarias fueron originalmente descriptos por Bougon en 1829. Se definen como dilataciones localizadas que exceden en 1,5 veces el diámetro del vaso adyacente normal. Pueden ser fusiformes o saculares, solitarios o múltiples ${ }^{3}$. A diferencia de los aneurismas, en los cuales la continuidad de la pared arterial está respetada, los pseudo-aneurismas se caracterizan por la ruptura de la pared arterial por lo que la sangre queda contenida sólo por la adventicia y los tejidos que rodean la arteria ${ }^{3}$. Ciertas características angiográficas permiten sospechar una u otra entidad, pero el diagnóstico diferencial solo puede confirmarse

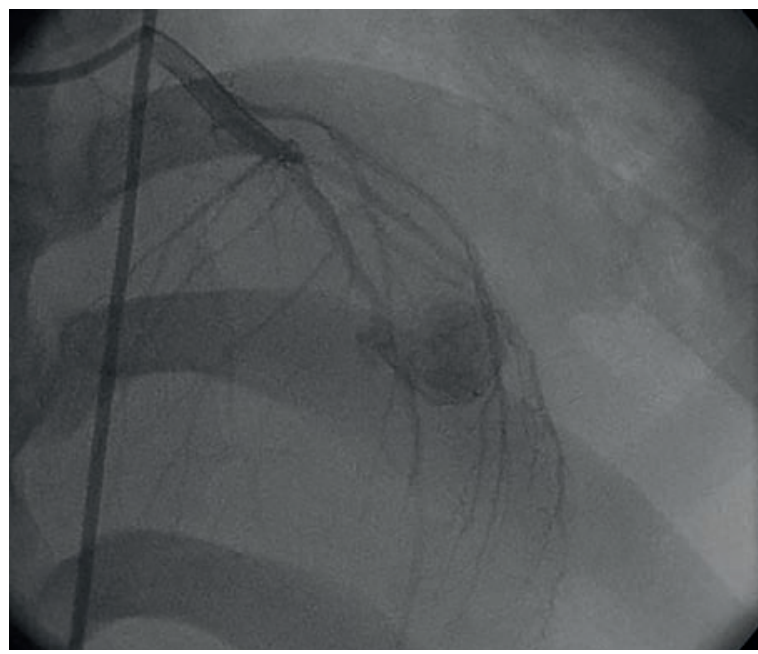

Figura 1. Pseudoaneurisma en tercio medio de arteria descendente anterior (proyección PA craneal $25^{\circ}$ ). 


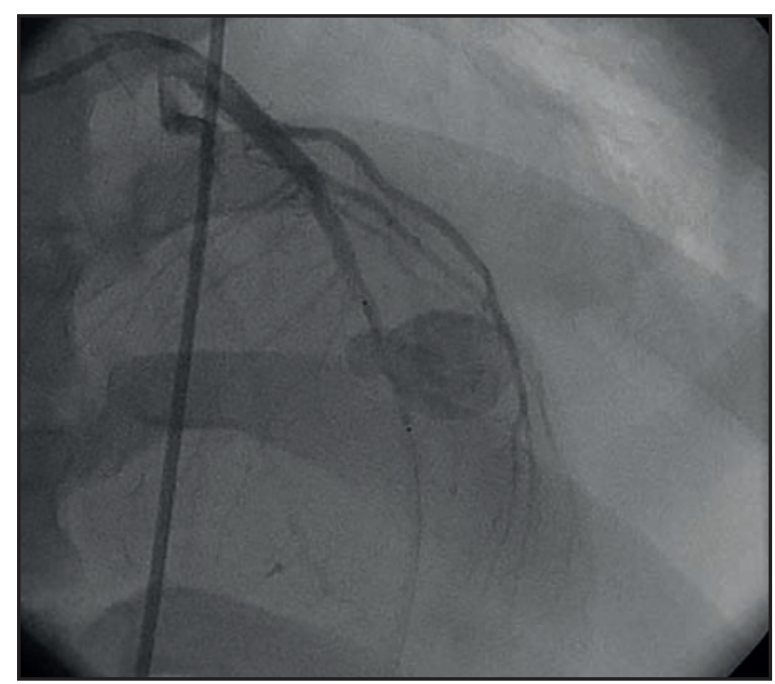

Figura 2. Pseudoaneurisma bilobulado con stent posicionado previo al implante.

mediante ultrasonido intravascular (Intra Vascular Ultra Sound, IVUS), la visualización directa en la sala de operaciones o por evaluación anátomo-patológica ${ }^{3}$. La incidencia de aneurismas en arterias coronarias es del 0,3\% al 4,9\% según diferentes publicaciones de angiografías y de autopsias; particularmente son raros aquellos localizados en el tronco de la coronaria izquier$\mathrm{da}^{9,10}$. La etiología más frecuente es la aterosclerosis, pero otras posibles causas incluyen la enfermedad de Kawasaki, lupus eritematoso sistémico, esclerodermia, síndrome de Marfán, periarteritis nodosa, arteritis de Takayasu, síndrome de Ehlers-Danlos, malformaciones congénitas, aneurismas micóticos y postraumáticos ${ }^{9,11}$. Los pseudoaneurismas, en cambio, son aún más infrecuentes y solo se han publicado casos aislados ${ }^{1-8}$. Ocurren principalmente como consecuencia de traumas originados por procedimientos percutáneos, disecciones espontáneas o traumatismos de tórax ${ }^{1-8}$. Si bien en los aneurismas la embolización del trombo allí formado y el consiguiente infarto es una complicación más frecuente que la ruptura, en los pseudo-aneurismas la ruptura es la complicación más temida ${ }^{9,11,12}$. Existe considerable controversia acerca del tratamiento más adecuado de estos pseudoaneurismas. Las opciones son el tratamiento conservador con agentes antiplaquetarios o anticoagulantes orales, el tratamiento percutáneo con coils o stents cubiertos y la corrección quirúrgica con reconstrucción, resección, exclusión de la

\section{BIBLIOGRAFÍA}

1. lemura J, Oku H, Shirotani H. Right coronary pseudoaneurysm after blunt injury to the chest. Heart 1996; 76: 86.

2. Daisuke Y, Hironori I, Masahiro R, Kanji K, and Yoshiki S. Asymptomatic Giant Traumatic Right Coronary Artery Pseudoaneurysm Caused by Sternal Fracture Ann Thorac Surg 2011;92:e33

3. Kishi K, Hiasa Y, Takahashi T. Delayed development of a giant coronary pseudoaneurysm after stent placement for chronic total occlusion. J Invasive Cardiol 2003; 15:273-6.

4. Rahman S, Abdul-Waheed M, Helmy T, et al. Spontaneous left main coronary artery dissection complicated by pseudoaneurysm formation in pregnancy: role of C $T$ coronary angiography. J Cardiothorac Surg 2009; 4: 15-8.

5. Wu IH, Koullias GJ, Dewar ML, et al. Hemodynamic compromise from a right coronary artery pseudoaneurysm after remote stent placement. Ann Thorac surg 2005; 79: 1062.

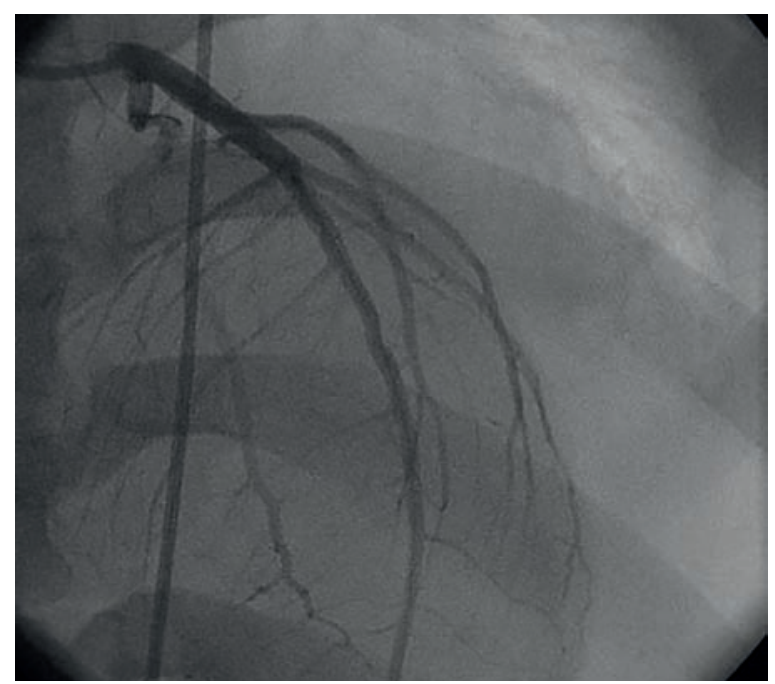

Figura 3. Posimplante de stent coronario cubierto de PTFE marca Be Graft (Bentley) de 3,0 mm de diámetro por $21 \mathrm{~mm}$ de longitud en tercio medio.

lesión o ambas, combinado con bypass de la coronaria comprometida $^{1-4,8,9}$. El tratamiento conservador es una estrategia frecuentemente utilizada en los aneurismas coronarios sobre todo en aquellos que no han embolizado o adquirido gran tamaño 9 . Con los pseudoaneurismas, en cambio, debido al mayor riesgo de ruptura, la mayoría de los casos publicados han sido tratados con procedimientos percutáneos o quirúrgicos ${ }^{1-4}$.

Creemos que nuestro paciente tiene un pseudoaneurisma, en lugar de un aneurisma, debido a lo digitiforme de la lesión y a su origen postraumático. No pudimos confirmarlo debido a que no se usó IVUS en el cateterismo realizado y a que no fue intervenido quirúrgicamente. El infarto antero-septal y apical probablemente fue causado por la embolización del trombo formado en el interior del pseudoaneurisma. Debido al elevado riesgo de ruptura de los pseudoaneurismas, la corrección percutánea fue una estrategia razonable. Creemos que los pseudoaneurismas deberían en general ser tratados con procedimientos percutáneos o quirúrgicos debido a su elevado riesgo de ruptura, aunque en ocasiones pueden permanecer estables en el tiempo con tratamiento conservador. Nuevos métodos radiológicos como la angiografía coronaria por tomografía computarizada multicorte son muy útiles para el seguimiento debido a que brindan en forma no invasiva imágenes precisas del tamaño y morfología de la lesión.
AquelRA,ZoghbiGJ,IskandrianAE. Sponteneouscoronaryarterydissection, aneurysms, and pseudoaneurysms: areview. Echocardiography 2004; 21: 175-82.

7. Manari A, Giacometti P, Vergoni W, et al. Acute myocardial infarction in pregnancy in a patient with pseudo-aneurysm of the left main coronary artery. $G$ Ital Cardiol 1996; 26: 1437-43 (Abstract).

8. Stone AL, Fleming HA. Aneurysm of left ventricle and left coronary artery after non-penetrating chest trauma Br Heart J 1983; 50: 495-7.

9. Lima B, Varma SK, Lowe JE. Nonsurgical management of left coronary artery aneurysms. Tex Heart Inst J 2006; 33: 376-9.

10. Swaye PS, Fisher LD, Litwin P, et al. Aneurysmal coronary artery disease. Circulation 1983; 67: 134-8.

11. Robinson FC. Aneurysm of the coronary arteries. Am Heart J 1985; 109: 129-35.

12. Rath S, Har-Zahav Y, Battler A, et al. Fate of nonobstructive aneurysmatic coronary artery disease: angiographic and clinical follow-up report. Am Heart J 1985; 109: 785-91. 